\title{
Aminopyridazines inhibit $\beta$-amyloid-induced glial activation and neuronal damage in vivo
}

\author{
Jeffrey M. Craft ${ }^{\mathrm{a}, \mathrm{b}}$, D. Martin Watterson ${ }^{\mathrm{a}, \mathrm{c}}$, Sally A. Frautschy ${ }^{\mathrm{d}}$, Linda J. Van Eldik ${ }^{\mathrm{a}, \mathrm{b}, *}$ \\ a Drug Discovery Program, Northwestern University Feinberg School of Medicine, Chicago, IL, USA \\ ${ }^{\mathrm{b}}$ Department of Cell and Molecular Biology, Northwestern University Feinberg School of Medicine, Chicago, IL, USA \\ ${ }^{\mathrm{c}}$ Departments of Molecular Pharmacology and Biological Chemistry, Northwestern University Feinberg School of Medicine, Chicago, IL, USA \\ ${ }^{\mathrm{d}}$ VAGLAHS-Sepulveda GRECC, Departments of Medicine and Neurology, UCLA, North Hills, CA, USA
}

Received 20 October 2003; received in revised form 23 December 2003; accepted 6 January 2004

\begin{abstract}
The critical role of chronic inflammation in disease progression continues to be increasingly appreciated across multiple disease areas, especially in neurodegenerative disorders such as Alzheimer's disease. We report that late intervention with a recently discovered aminopyridazine suppressor of glial activation, developed to inhibit both oxidative and inflammatory cytokine pathways, attenuates human amyloid beta $(A \beta)$-induced glial activation in a murine model. Peripheral administration of the aminopyridazine MW01-070C, beginning 3 weeks after the start of intracerebroventricular infusion of human A $\beta 1-42$, decreased the number of activated astrocytes and microglia and the levels of proinflammatory cytokines interleukin-1 $\beta$, tumor necrosis factor- $\alpha$ and S100B in the hippocampus. Inhibition of neuroinflammation correlated with a decreased neuron loss, restoration towards control levels of synaptic dysfunction biomarkers in the hippocampus, and diminished amyloid plaque deposition. The results from this in vivo chemical biology approach provide a proof of concept that targeting of key glia inflammatory cytokine pathways can suppress $A \beta$-induced neuroinflammation in vivo, with resultant attenuation of neuronal damage.

(C) 2004 Elsevier Inc. All rights reserved.
\end{abstract}

Keywords: Alzheimer's disease; Neuroinflammation; Glia; Drug discovery; Animal model; Aminopyridazine; Amyloid; Hippocampus

\section{Introduction}

The contribution of the inflammatory component of diverse disorders to disease progression has led to novel attempts aimed at discovering ways to attenuate inflammation therapeutically [21]. Key among these attempts are efforts to modulate the neuroinflammation that is a characteristic feature of both acute and chronic CNS disorders $[1,18,22]$. Neuroinflammation is a process that results primarily from an abnormally high or chronic activation of glia (microglia and astrocytes). This overactive state of glia results in increased levels of inflammatory and oxidative stress molecules, which can lead to neuron damage or death. Neuronal damage/death can also induce glial activation, facilitating the propagation of a localized, detrimental cycle of neuroinflammation [8]. Accumulating evidence [1,6,9,13] suggests that targeting this glia-neuron cycle might be a therapeutic approach to Alzheimer's disease (AD) progression. However, progress in the pursuit of neuroinflammation as a therapeu-

\footnotetext{
* Corresponding author. Tel.: +1-312-503-0697; fax: +1-312-503-0007.
}

E-mail address: vaneldik@northwestern.edu (L.J.V. Eldik). tic target in $\mathrm{AD}$ requires proof of concept that selective suppressors of glial activation can selectively modulate neuropathogenic aspects of the neuroinflammatory cycle, without impeding beneficial glial responses, in a robust animal model of AD-relevant neuroinflammation. In general, validation in an $\mathrm{AD}$ animal has been difficult due to the variability of neuroinflammation and the lack of frank neuronal loss in the animal models, and the few compounds tested to date were not developed with glia as the primary discovery target.

MW01-070C is an aminopyridazine derivative discovered in unbiased cell-based screens for new synthetic compounds that suppress selective glial activation responses via mechanisms distinct from anti-inflammatories developed for peripheral tissue targets, such as NSAIDs and COX-2 inhibitors. Aminopyridazines with activities similar to MW01-070C have the potential to fulfill the unmet need for modulation of AD-relevant neuroinflammation. The discovery rationale was to find safe compounds capable of suppressing pathways that are quantitatively important in proinflammatory and oxidative stress responses of activated glia, versus a primary focus on suppression of peripheral tissue responses. The initial screen with a high 
throughput, cell-based assay included comparison to extant anti-inflammatory drugs in order to find new synthetic compounds with significantly better, concentration-dependent activity for inhibition of activated glia responses $[16,17,35]$. A secondary cell-based screen was employed to retain only those compounds that worked via mechanisms distinct from currently available NSAIDs that target COX-2 or p38 MAPK, and to find compounds that would not suppress potential anti-inflammatory responses of glia, such as increased ApoE production in response to $A \beta 1-42$ stimulation. This cell-based, hierarchical approach held the potential of finding safe compounds that would effectively suppress disease-relevant endpoints in glia, but would not suppress the immune response in peripheral tissues, which are needed for responses to infections. MW01-070C fulfills the above criteria based on cell-based activity analyses and initial toxicology studies [17,35]. However, MW01-070C has not been validated in vivo using an animal model of AD-relevant neuroinflammation.

We report here that peripheral administration of MW01070C, beginning 3 weeks after start of intracerebroventricular infusion of human A $\beta 1-42$ into mouse brain, attenuates the resultant neuroinflammation and neuronal loss. We used a rapid and reproducible in vivo assay for human $\mathrm{A} \beta$-induced neuroinflammation in mice, based on the previously described rat model of Frautschy et al. [6,7]. The infusion of human $A \beta 1-42$ increased the number of activated astrocytes and microglia and the levels of interleukin- $1 \beta$ (IL-1 $\beta$ ), tumor necrosis factor- $\alpha(\mathrm{TNF} \alpha)$ and S100B in the hippocampus. In addition, the mice showed a decreased neuronal count and a decrease in markers of synaptic function in the hippocampus. These changes were attenuated by therapy with MW01-070C, administered intraperitoneally and initiated 3 weeks after the start of human $A \beta$ infusion. The results demonstrate the therapeutic potential of targeting of glial inflammatory pathways with aminopyridazines.

\section{Methods}

\subsection{A $\beta$ infusion into the CNS and treatment with inhibitor by intraperitoneal injection}

The study design and treatment paradigm for infusion of human $A \beta 1-42$ into the mouse was adapted from a rat model of human $A \beta$ infusion [6,7]. Briefly, C57/B16 mice weighing $\sim 25 \mathrm{~g}$ were anesthetized with vaporized halothane, and a micro-osmotic pump (Alzet \#1002) was attached to a cannula (Plastics One) stereotaxically implanted into the right lateral cerebral ventricle (at coordinates $-1.0 \mathrm{~mm}$ mediolateral and $-0.5 \mathrm{~mm}$ anterioposterior from Bregma; $-1.5 \mathrm{~mm}$ dorsal-ventral from skull). Pumps contained either oligomeric [4] A $\beta 1-42$ (45 $\mu \mathrm{g}$; American Peptide) in vehicle $(4 \mathrm{mM}$ Hepes $+250 \mu \mathrm{g} / \mathrm{ml}$ human high-density lipoprotein, HDL) or vehicle alone. HDL, which normally carries $A \beta$ in plasma, was used in the pump to reduce $A \beta$ aggregation and act as an $\mathrm{A} \beta$ chaperone for better neuropil delivery [6,7]. Pumps were partially coated with paraffin to reduce the infusion rate to $\sim 3 \mu \mathrm{l}$ per day $(67 \mathrm{ng} / \mathrm{h} \mathrm{A} \beta 1-42$; $37 \mathrm{ng} / \mathrm{h} \mathrm{HDL}$ ) for 28 days.

At post-operative day 21 and continuing for 14 days thereafter, mice were injected once a day with an intraperitoneal injection of either MW01-070C (2.5 mg/kg per day) or solvent control (10\% DMSO in saline). The dose of MW01-070C represents a maximal dose for this experimental paradigm based on results that showed suppression of IL-1 $\beta$ levels to that of control (vehicle), and a corresponding intermediate level of suppression with one-half the dose of MW01-070C. In some experiments, a structurally similar pyridazine-based compound, MW01-026Z, was injected intraperitoneally at $2.5 \mathrm{mg} / \mathrm{kg}$ exactly as described for MW01-070C. At post-operative day 60, mice were anesthetized with pentobarbital $(50 \mathrm{mg} / \mathrm{kg})$ and perfused with a Hepes buffer containing a protease inhibitor cocktail. The brains were then removed and longitudinally bisected. The right half of the brain was fixed in paraformaldehyde and embedded in paraffin for histological examination, while the hippocampus was isolated from the left hemisphere and snap frozen for biochemical evaluation.

MW01-070C, 11-(5,6-dihydro-3-iminobenzo[h]cinnolin2(3H)-yl)-1-(4-pyrimidin-2-yl-piperazin-1-yl)undecanamide, was synthesized and characterized as previously described [17]. MW01-026Z, 11-(5,6-dihydro-3-iminobenzo[h] cinnolin-2(3H)-yl)- $N$-(6-phenylpyridazin-3-yl)undecanamide, is a structural analog of MW01-070C and was synthesized and characterized as previously described [33].

\subsection{Biochemical analysis of inflammatory and neural markers in the hippocampus}

Hippocampal soluble extracts were prepared by dounce and sonication in Hepes buffer containing a protease inhibitor cocktail, followed by centrifugation. Levels of the pro-inflammatory cytokines IL- $1 \beta$ and $\mathrm{TNF} \alpha$ in supernatants were determined with duplicate measurements by a Biosource International ELISA according to the manufacturer's instructions. S100B levels in supernatants were measured in triplicate by a europium-based ELISA essentially as previously described [32]. Synaptophysin levels were quantified in duplicate with a capture ELISA following the procedure previously described by Schlaf et al. [27] with the following exceptions. The SY38 monoclonal synaptophysin antibody (Chemicon International) was used to coat plates at the concentration of $1.75 \mu \mathrm{g} / \mathrm{ml}$, while a rabbit polyclonal synaptophysin antibody $(0.35 \mu \mathrm{g} / \mathrm{ml}$, Calbiochem $)$ and a horseradish root peroxidase (HRP)-conjugated goat anti-rabbit IgG antibody $(1: 10,000$, Jackson Immunolabs) were used for detection with a tetramethylbenzidine (TMB) development system (Sigma). Due to the lack of purified synaptophysin, a cortico-hippocampal homogenate was prepared in the same manner as the hippocampal soluble extracts to serve as a 
standard. Western blots of hippocampal supernatants $(10 \mu \mathrm{g}$ supernatant protein loaded per lane) were done with antibodies to PSD-95 (1:100,000 dilution; Upstate Biotechnology) and COX-2 (1:2000 dilution, Santa Cruz). Antibodies against $\beta$-actin (1:500,000 dilution, Sigma) were used to confirm equal protein loading among the samples. Densitometry was done with ImageQuant software (Molecular Dynamics).

\subsection{Histology}

Immunohistochemical detection of activated astrocytes and microglia was performed on $10 \mu \mathrm{m}$ sections with anti-GFAP (1:1500 dilution; Sigma) and anti-F4/80 (1:100 dilution; Serotek) antibodies, respectively, using the mouse on mouse or Vectastain Universal Elite ABC immunodetection kits (Vector/Novocastra) and development with diaminobenzidine (DAB) substrate. $A \beta$ immunohistochemistry was done with DAE (rabbit anti-human $A \beta$ antibody; [13]; kindly provided by Greg Cole, UCLA) using the Vectastain Rabbit Elite ABC kit (Vector/Novocastra) and development with TrueBlue Substrate (KPL). Staining of brain architecture and neurons was carried out with $0.2 \%$ cresyl violet acetate solution. Cell counts, plaque counts, and $A \beta$ load were determined by two blinded observers and subsequently analyzed as follows. For microglia and astro- cyte analysis, all DAB-stained cell bodies were manually counted in the hippocampus (excluding the fimbria) of three F4/80 and GFAP labeled sections positioned at $-1.8,-2.1$, and $-2.3 \mathrm{~mm}$ from Bregma. For plaque counts and $A \beta$ load analysis, images of five equally spaced DAE labeled sections from approximately $-1.4 \mathrm{~mm}$ through $-2.4 \mathrm{~mm}$ from Bregma were digitally obtained and examined using Image $\mathrm{J}$ software. Plaque number was determined by manually counting the number of plaques on each section, and the average number of plaques per section was then calculated. Plaque area was determined by outlining the boundaries of all plaques on each section and obtaining the summed area with Image $\mathbf{J}$ software (NIH public domain software). The plaque area was then divided by the area of the section to determine percent amyloid staining on each section, and then the average percent amyloid staining per section was calculated. CA1 neuron counts were obtained by digitally acquiring images (200× magnification) of the CA1 neuron layer immediately superior to the dentate gyrus of multiple cresyl violet stained sections spaced $50 \mu \mathrm{m}$ apart from $-1.2 \mu \mathrm{m}$ through $-2.4 \mathrm{~mm}$ from Bregma (20-25 sections per animal). Blinded observers then differentiated neurons from glia by cellular morphology and size and counted only neurons with nuclei present in the section. In all studies, concordance between observers was within $5 \%$ or the section was removed from analysis.

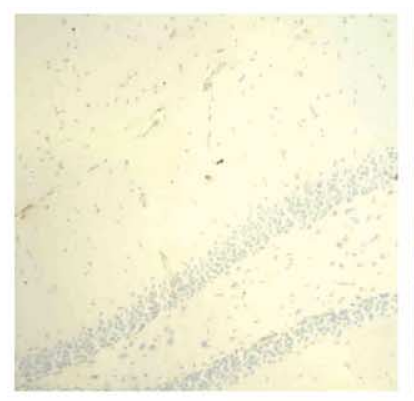

(A)

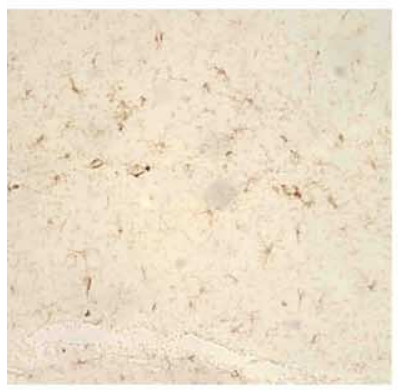

(E)

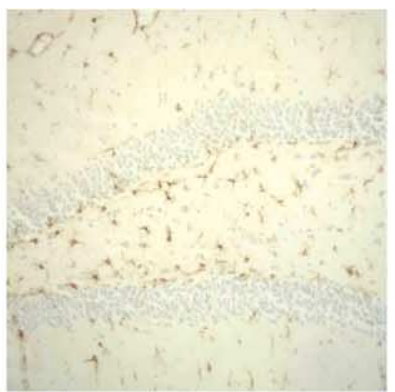

(B)

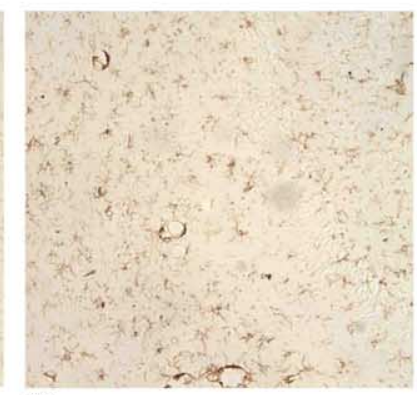

(F)

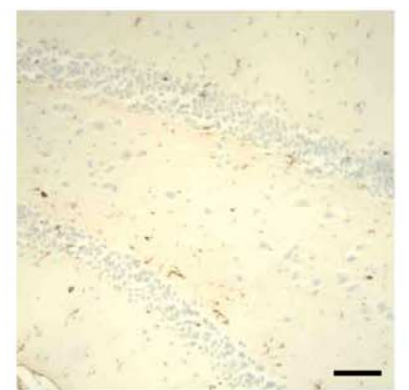

(C)

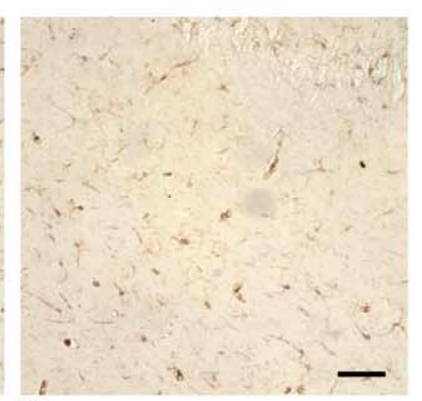

(G)

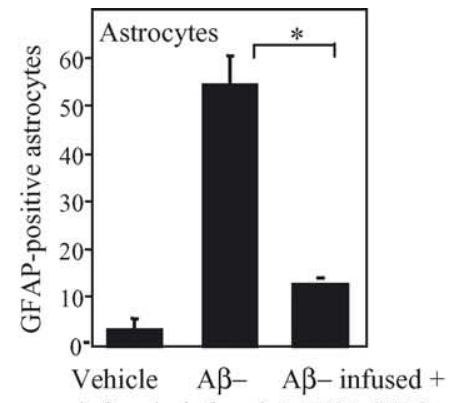

(D)

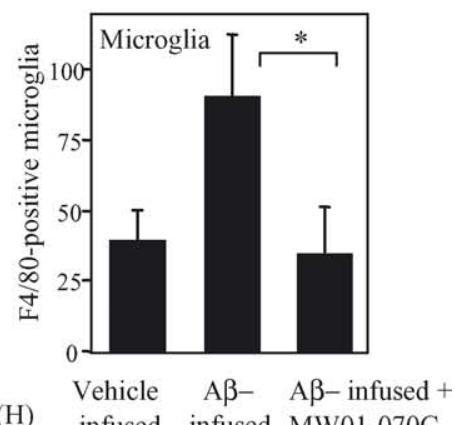

Fig. 1. MW01-070C suppresses astrocyte and microglial activation. (A-D) Activated astrocytes were detected by GFAP immunoreactivity, and the number of GFAP-positive astrocytes in the hippocampus were measured. Representative micrographs of vehicle-infused mice (A), A $\beta$-infused mice injected with solvent (B), and A $\beta$-infused mice injected with MW01-070C (C) are shown. Bar (A-C) $=50 \mu \mathrm{m}$. Quantitation of GFAP-positive astrocytes from all mice ( $n=5-8$ mice per group) is shown (D). (E-H) Activated microglia were detected by F4/80 immunoreactivity, and the number of F4/80-positive microglia in the hippocampus were measured. Representative micrographs of vehicle-infused mice (E), A $\beta$-infused mice injected with solvent (F), and A $\beta$-infused mice injected with MW01-070C $(\mathrm{G})$ are shown. Bar $(\mathrm{E}-\mathrm{G})=25 \mu \mathrm{m}$. Quantitation of F4/80-positive microglia from all mice $(n=5 \mathrm{mice}$ per group) is shown $(\mathrm{H})$. Significantly different $\left({ }^{*} P<0.05\right)$. 


\subsection{Statistical analysis}

Experimental and control groups were compared by ANOVA with SNK post hoc analysis (significant at $P<$ $0.05)$.

\section{Results}

\subsection{In vivo glial activation by human $A \beta$ and suppression by aminopyridazine treatment}

Intracerebroventricular infusion of mice with human A $\beta 1-42$ for 28 days and analysis of hippocampal tissue harvested at day 60 showed, by several biochemical and histological endpoints, robust neuroinflammation. This neuroinflammation was suppressed by late stage intervention with MW01-070C at a remote site (intraperitoneal injection) starting at day 21. Activation of astrocytes and microglia in the hippocampus is seen by glial fibrillary acidic protein (GFAP) and F4/80 immunohistochemistry, respectively (Fig. 1). There were significantly greater numbers of GFAPpositive astrocytes in the hippocampus of mice infused with $A \beta$ versus those infused with vehicle, and peripheral administration of MW01-070C attenuated this astrocyte activation (Fig. 1A-D). There was a similar suppressive effect of inhibitor treatment on microglial activation (Fig. 1E-H). Administration of MW01-070C alone did not result in any detectable glial activation (data not shown).

\subsection{Selective suppression of neuroinflammatory mediators} by aminopyridazine treatment 3 weeks after initiation of $A \beta$ infusion

The $A \beta$-induced activation of astrocytes and microglia detected by immunohistochemical analysis of mouse brain sections was reflected in increased production of AD-relevant pro-inflammatory cytokines measured by quantitative immunochemical endpoints (Fig. 2). Initiation of MW01-070C treatment 21 days after the start of $A \beta$ infusion into the brain, using administration of MW01-070C outside the CNS (intraperitoneal injection), resulted in a significant and selective reduction of pro-inflammatory cytokine levels in the hippocampus (Fig. 2). For example, we found that mice infused with $A \beta 1-42$ showed increases in IL-1 $\beta$ levels, which were attenuated by administration of MW01-070C (Fig. 2A). Similarly, the levels of the pro-inflammatory cytokine TNF $\alpha$ were significantly upregulated in the hippocampus of $A \beta-$ infused animals (Fig. 2B), while peripheral administration of MW01-070C suppressed the A $\beta$-induced TNF $\alpha$ to levels statistically indistinguishable from those mice receiving vehicle infusion (Fig. 2B). We also found that the levels of the pro-inflammatory cytokine S100B in the hippocampus were significantly higher in mice receiving human $A \beta$ infusion (Fig. 2C), with intraperitoneal administration of MW01-070C subsequent to the initiation of increased $A \beta$
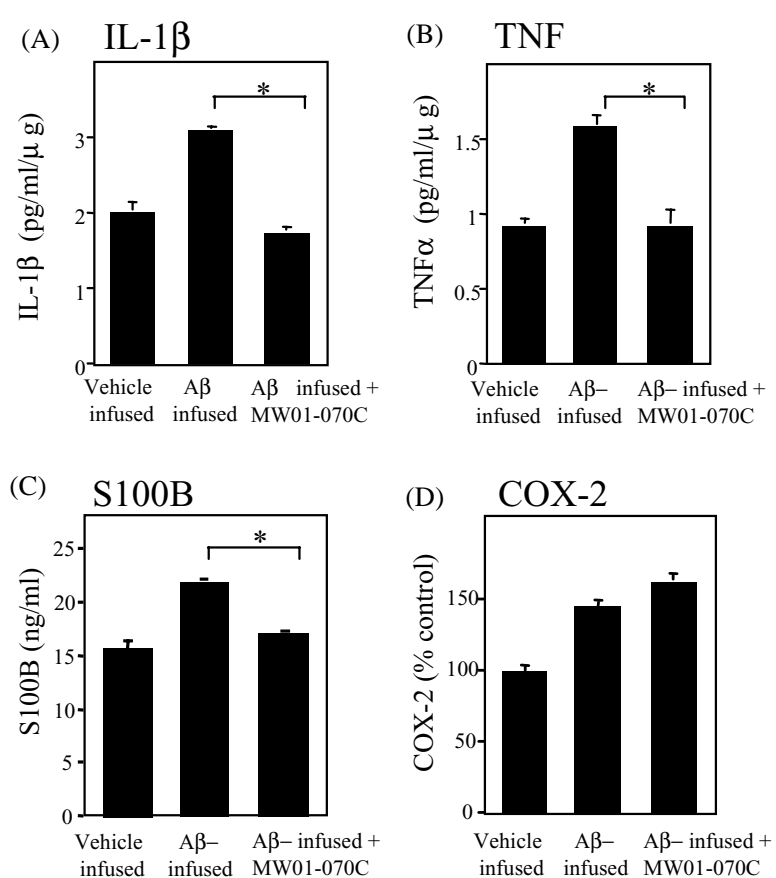

Fig. 2. MW01-070C selectively suppresses neuroinflammatory responses. The levels of the pro-inflammatory cytokines IL-1 $\beta$ (A), TNF $\alpha$ (B), S100B (C), and COX-2 (D) were measured in hippocampal extracts from vehicle-infused mice, $\mathrm{A} \beta$-infused mice injected with solvent, and $\mathrm{A} \beta$-infused mice injected with MW01-070C ( $n=5-8$ mice per group). $\mathrm{A} \beta$ induced a significant increase in all four cytokines. MW01-070C treatment resulted in significant suppression of the increase in IL-1 $\beta$, TNF $\alpha$, S100B, but not COX-2. Significantly different $\left({ }^{*} P<0.05\right)$.

in the brain resulting in a significant reduction of S100B levels in the hippocampus (Fig. 2C). The therapeutic effects of peripherally administered MW01-070C on AD-relevant neuroinflammatory markers in the hippocampus were complemented by the observation of in vivo selectivity in pharmacological action. Specifically, the $A \beta$-induced increase in COX-2 levels in the hippocampus was not blocked by MW01-070C treatment (Fig. 2D). This selective in vivo effect of MW01-070C is consistent with its lack of effect on COX-2 levels in cultured cells [17].

\subsection{Attenuation of neuronal loss by in vivo treatment with a selective suppressor of glial activation}

An important disease-relevant endpoint to monitor is whether the in vivo suppression of neuroinflammation by a selective inhibitor of glial activation results in significant attenuation of neuronal function or loss. Therefore, we examined neuron-related endpoints in the mouse hippocampus. First, we examined neuron number by cresyl violet staining of serial sections throughout the hippocampus. We found that neuron number in the CA1 region of the hippocampus was significantly decreased in $A \beta$-infused mice (Fig. $3 \mathrm{~A}$ ). However, $A \beta$-infused mice treated intraperitoneally with MW01-070C were indistinguishable from control animals 

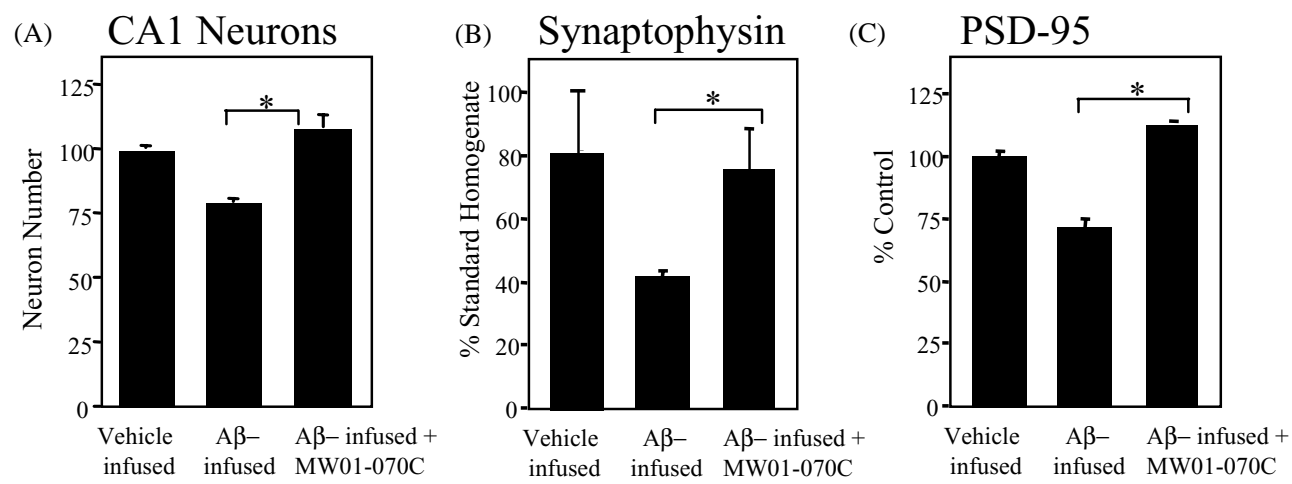

Fig. 3. MW01-070C blocks neuronal damage and death. Hippocampal sections or extracts from vehicle-infused mice, Aß-infused mice injected with solvent, and $\mathrm{A} \beta$-infused mice injected with MW01-070C ( $n=5-8$ mice per group) were evaluated for neuron damage/death by determination of the number of hippocampal CA1 neurons (A), levels of synaptophysin by ELISA (B), and levels of the post-synaptic density protein PSD-95 by Western blots $(\mathrm{C})$. Significantly different $\left({ }^{*} P<0.05\right)$.

in CA1 neuron number (Fig. 3A). These histological results were corroborated by biochemical measurements of two different neuronal markers. Determination of the levels of the presynaptic protein synaptophysin by ELISA showed that $A \beta$ infusion led to a significant reduction in synaptophysin, and the decrease in synaptophysin levels was prevented by MW01-070C administration (Fig. 3B). The effect seen with the pre-synaptic marker protein was also seen with a post-synaptic marker. Specifically, Western blot analysis of post-synaptic density protein-95 kDa (PSD-95) showed significant reduction in $\mathrm{A} \beta$-infused mice (Fig. 3C). PSD-95 is a major post-synaptic component of excitatory glutaminergic synapses that are reduced in brain injuries that result in spatial memory deficits [15]. In contrast, $A \beta$-infused
(A)
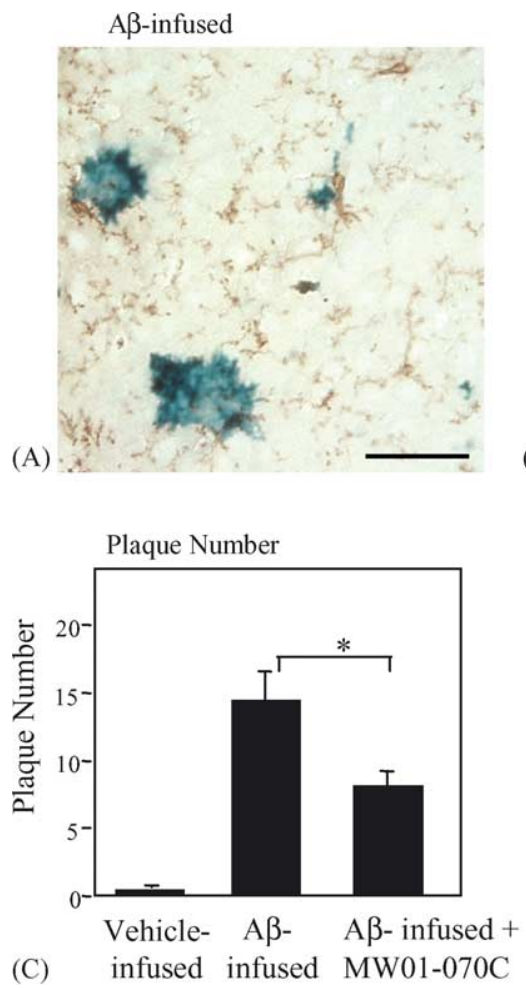

$\mathrm{A} \beta+\mathrm{MW01-070C}$
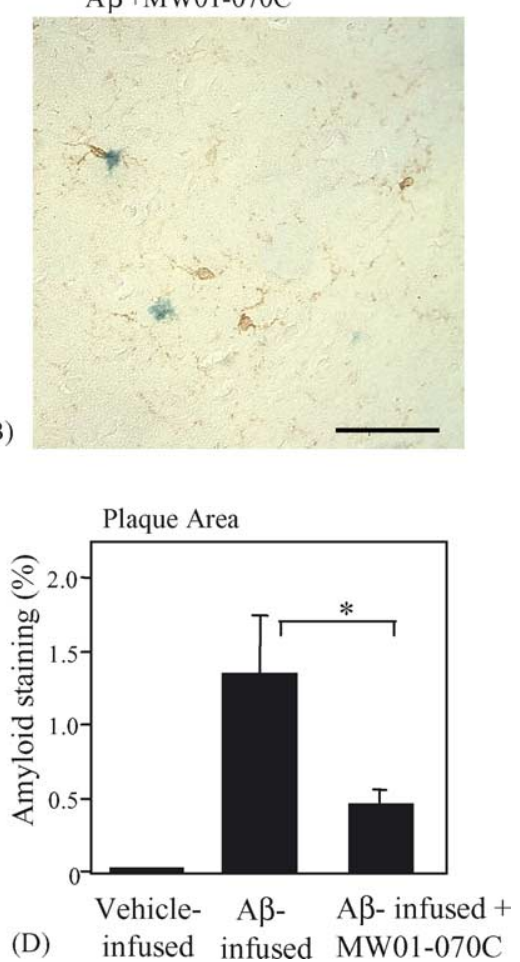

Fig. 4. MW01-070C reduces amyloid plaque deposition. Hippocampal sections from vehicle-infused mice, A $\beta$-infused mice injected with solvent, and A $\beta$-infused mice injected with MW01-070C were double-labeled as described in Section 2 with anti-human A $\beta 1-42$ antibody to stain amyloid plaques (blue) and with anti-F4/80 antibody to stain activated microglia (brown). Representative micrographs of hippocampus from $A \beta$-infused mice injected with solvent (A), and A $\beta$-infused mice injected with MW01-070C (B) are shown. Bar $=10 \mu \mathrm{m}$. Quantitation of amyloid burden from all mice $(n=5-8$ mice per group) was done as described in Section 2 by determination of the number of amyloid plaques (C) and the area occupied by amyloid plaques (D). Significantly different $(* P<0.05)$. 
mice treated with MW01-070C showed PSD-95 levels similar to vehicle-infused animals (Fig. 3C). The results using multiple endpoints of hippocampal neuronal status are consistent with each other and demonstrate in this mouse model that human $A \beta$ infusion results in neuronal loss and intraperitoneal treatment with MW01-070C provides neuroprotection. This in vivo protection against neuronal damage afforded by MW01-070C treatment is consistent with its established activity as a selective inhibitor of neurotoxic glial products [17].

\subsection{Effects of inhibiting glial activation in vivo on $A \beta$ plaques}

In addition to the direct detrimental effects of activated glial products on neurons, certain glial products can influence $A \beta$ structure, function, and synthesis [1]. This provides an additional, indirect mechanism whereby suppression of glial activation could potentially attenuate $A \beta$ plaque growth

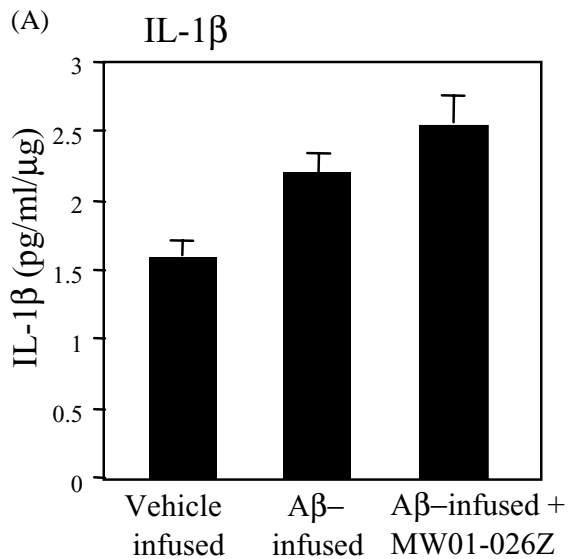

or toxic $A \beta$ aggregate formation. As an initial step in addressing this more indirect neuroprotective consequence of MW01-070C suppression of glial activation, we examined the amyloid plaques in our mouse model. Staining of hippocampal sections with an antibody that recognizes human $\mathrm{A} \beta$ (but not the endogenous mouse $\mathrm{A} \beta$ ) demonstrated diffuse amyloid plaque deposits in the hippocampus (Fig. 4A). In the $A \beta$-infused mice injected with MW01-070C, the amyloid plaques were smaller and less abundant (Fig. 4B). Quantitation of the numbers of plaques (Fig. 4C) and the area of the hippocampus occupied by amyloid plaques (Fig. 4D) documented a significant reduction in amyloid plaque deposition as a result of MW01-070C treatment.

\subsection{A CNS active structural analog of MW01-070C does not suppress neuroinflammation}

MW01-026Z is an aminopyridazine analog of MW01070C that does not have the glial suppression activity of
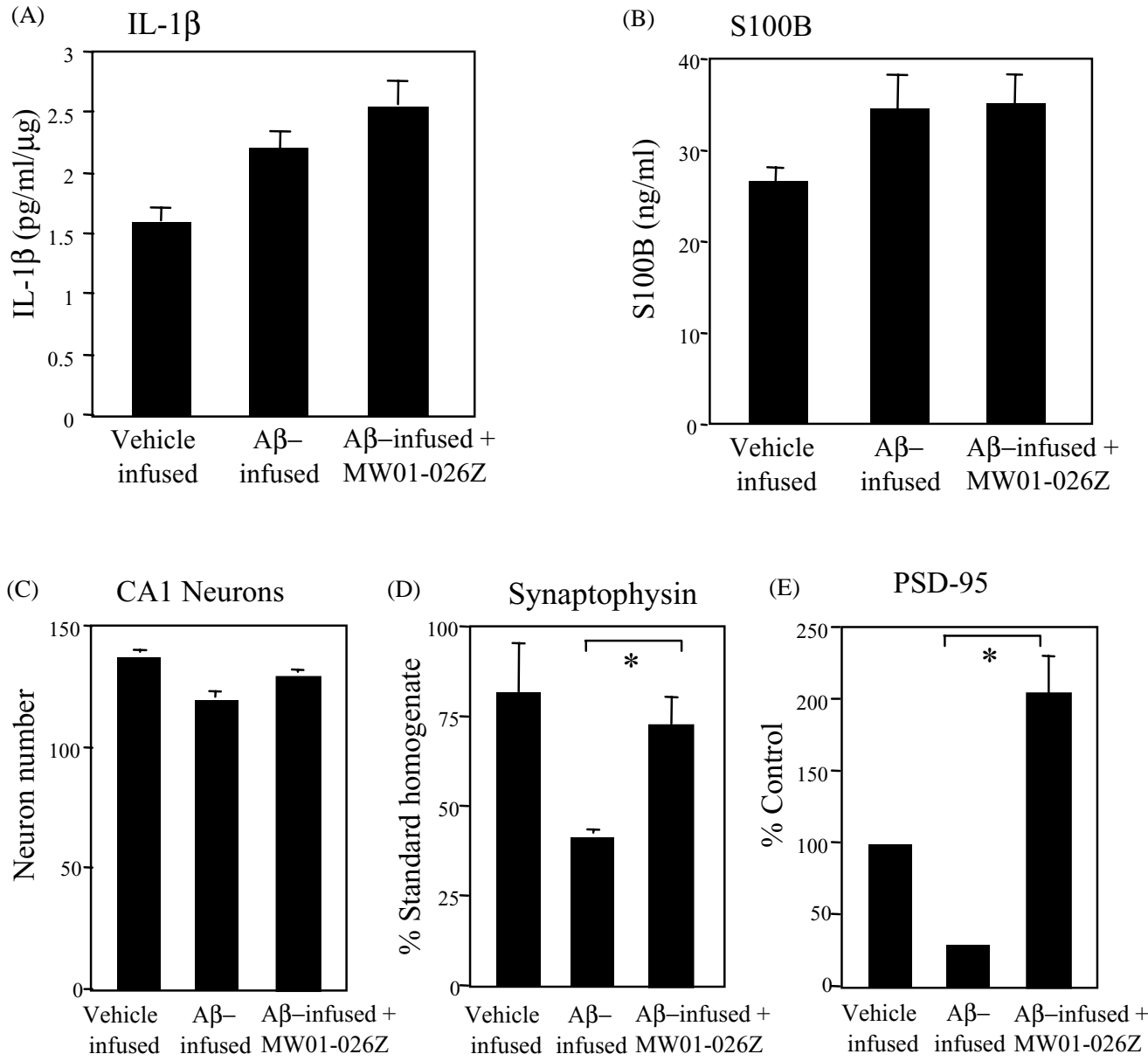

Fig. 5. MW01-026Z, a structural analog of MW01-070C that does not possess in vitro glial inhibitory activity but does provide neuroprotection in an acute brain injury model, does not inhibit neuroinflammation but protects neurons following $A \beta$ infusion. Measurement of the pro-inflammatory cytokines IL-1 $\beta$ (A) and S100B (B) reveal no attenuation of A $\beta$-induced glial activation. MW01-026Z partially protected neurons as determined by neuron counts in the CA1 layer of hippocampus (C), and significantly ameliorated the A $\beta$-induced reduction in the presynaptic marker synaptophysin as measured by ELISA (D). Interestingly, MW01-026Z treatment led to increased levels of the post-synaptic maker PSD-95 by $\sim 2$-fold over vehicle-infused animals (E). Significantly different $\left({ }^{*} P<0.05\right)$. 
MW01-070C, but is able to inhibit brain injury and neuronal loss in a rodent stroke model in which MW01-070C lacks activity [33]. Therefore, MW01-026Z is a structural analog of MW01-070C that has demonstrated CNS penetrance but does not suppress glial activation in cell culture. When MW01-026Z was administered using the same treatment paradigm as that used for MW01-070C, MW01-026Z failed to suppress glial activation in vivo. For example, the $\mathrm{A} \beta$-induced increases in hippocampal IL-1 $\beta$ (Fig. 5A) and S100B (Fig. 5B) were not blocked by MW01-026Z. In addition, the $A \beta$ increase in the number of activated astrocytes and microglia in the hippocampus was not significantly inhibited by MW01-026Z treatment, as measured by immunohistochemical analysis of GFAP-positive astrocytes and F4/80-positive microglia. The number of GFAP-positive astrocytes in vehicle-infused mice, $A \beta$-infused mice, and $A \beta$ infused mice injected with MW01-026Z were, respectively, $7.56 \pm 0.96,36.29 \pm 8.41$, and $25.21 \pm 2.39$ (mean \pm S.E.M.). The number of F4/80-positive microglia in vehicle-infused mice, $A \beta$-infused mice, and $A \beta$-infused mice injected with MW01-026Z were, respectively, 3.33 $\pm 0.94,42.97 \pm 7.43$, and $25.73 \pm 4.55$ (mean \pm S.E.M.). There was a trend toward a suppression of amyloid plaque deposition with MW01-026Z treatment, but the results did not reach statistical significance (mean $(\%)$ area staining \pm S.E.M. in vehicle-infused mice was $0.004 \pm 0.004$; in $\mathrm{A} \beta$-infused mice, $1.31 \pm 0.37$; and in $A \beta$-infused mice injected with MW01-026Z,0.78 \pm 0.19 ). Interestingly, administration of this neuronal apoptosis inhibitor MW01-026Z did suppress the $A \beta$-induced neuronal damage. For example, the $A \beta$-induced reduction in CA1 neuron number was partially prevented by MW01-026Z administration (Fig. 5C). MW01-026Z also significantly prevented the $A \beta$-induced decrease in synaptophysin levels (Fig. 5D). Unexpectedly, MW01-026Z treatment not only prevented the A $\beta$-induced decrease in PSD-95 levels but also led to a significantly higher level of PSD-95 in the hippocampus when compared to vehicle-infused animals (Fig. 5E). These serendipitous findings have not been pursued further as part of this investigation on neuroinflammation. Regardless, the results with MW01-026Z and MW01-070C treatments demonstrate that the in vivo suppression of neuroinflammation in the mouse model reflects well the in vitro glial suppression activity of aminopyridazines, and is not a generalized effect of treatment with a CNS active aminopyridazine compound.

\section{Discussion}

The results summarized here have three key aspects. First, they provide a precedent for AD drug discovery by demonstrating that de novo inhibitor development targeting selective glia activation pathways can yield bioavailable compounds that function in vivo as anti-neuroinflammatory and neuroprotective compounds. Second, the results indicate that proinflammatory cytokines might be useful biomarkers for monitoring $\mathrm{A} \beta$-induced neuroinflammation and response to therapeutic intervention. Third, an animal model that exhibits $A \beta$-induced neuroinflammation and neuronal loss is described, and this model responds to therapeutic intervention targeted to glial activation pathways or neuronal death pathways.

The urgent need for novel therapeutic strategies in the treatment of $\mathrm{AD}$ is apparent with the realization that the only currently approved therapies for AD are palliative in nature. Major efforts have gone into testing drugs approved for other disease applications as potential therapies for $\mathrm{AD}$ progression. Success to date has been limited and, in some cases, there are legitimate concerns over safety in chronic use $[2,10,11,23]$. There is no a priori reason to expect that a drug approved by the FDA will work in another disease area unless there is a common mechanism of disease involved. In addition, an FDA approved drug is not necessarily safer than a new drug developed explicitly for the target disease, as relative toxicity or therapeutic index is always a consideration in use. However, these more straightforward tests must be pursued because $\mathrm{AD}$ is a disease with major unmet needs and increasing impact. Clearly, multiple and complementary approaches must be tried in parallel. We chose to take a high risk approach that lacked precedent, but was based on the established drug discovery paradigm of biological screening using disease-relevant endpoints, followed by analysis of biological novelty and potential for safety. We focused on glial activation and used in-parallel synthetic chemistry and hierarchal cell-based screens of disease-relevant endpoints. We also biased the process towards effective inhibitors that worked via mechanisms distinct from drugs that work on peripheral tissue inflammation. Eukaryotic cells can use diverse signal transduction pathways to modulate a redundant biological endpoint, with tissue context reflecting which pathway is quantitatively dominant. For example, iNOS and IL- $1 \beta$ are produced by a variety of cell types, but the relative importance of a given pathway to these same endpoints can vary among tissues. This might be the explanation for inhibition by MW01-070C of endotoxin-induced glial production of IL-1 $\beta$, with no discernible effect over the same concentration range of MW01-070C on endotoxin-induced production of IL- $1 \beta$ by peripheral blood monocytes. Much remains to be done in terms of elucidating the mechanisms of action of MW01-070C and newer analogs that are currently under development. However, the results presented here and previously $[17,35]$ validate the potential utility of the glia-focused approach in AD drug discovery.

We chose the aminopyridazine chemotype as our scaffold based on its prior use in development of clinically effective and safe drugs for chronic use $[14,36]$. The results presented here and previously $[17,34,35]$ identify aminopyridazines as a new chemotype for neuroinflammation drug discovery, and partially validate proinflammatory cytokines and iNOS as key glial activation pathways that are amenable to therapeutic intervention with aminopyridazines. Further, the successful intervention with an aminopyridazine 3 weeks after 
the start of $A \beta$-infusion argues in favor of pursuing these pathways and this small molecule class if late stage intervention in neuroinflammation is the goal. The attenuation of neuronal loss by an aminopyridazine developed for inhibition of glia activation, and by a structural analog developed for inhibition of neuronal apoptosis, is indicative of the potential for targeting either glia or neuronal responses for protection from human $A \beta$-induced pathology. Potentially, such targeted compounds may be useful reagents for in vivo chemical biology studies that seek to quantify the relative importance of a given pathway versus another to a common pathology endpoint.

The extent of in vivo glial activation by $\mathrm{A} \beta$ and its suppression by the aminopyridazine were assessed by quantitative analysis of hippocampal cytokine levels, including a cytokine readily measured in human CSF. This raises the possibility of identifying clinical biomarkers that are indicators of both neuroinflammation and response to therapeutic intervention. S100B is a pro-inflammatory cytokine that is increased in brain parenchyma and CSF of AD patients, raising the potential that this cytokine contributes to the neuroinflammation cycle or might be a biomarker for disease progression [20,24,25,31]. Recent research also showed that S100B levels in CSF can be used as an indicator of clinical outcome (for references, see [29,32]). Biological endpoints that can be readily transferred from preclinical studies with animals to clinical investigations are critical to translation of promising preclinical findings to new therapeutic approaches to CNS disorders $[3,28,30]$. Therefore, an important future goal is to validate the potential to monitor increases of S100B levels with $\mathrm{AD}$ disease progression and decreases in S100B levels with pharmacologic intervention. Relatedly, the finding that aminopyridazine treatment blocks hippocampal CA1 neuron loss suggests the need to investigate volume changes using MRI analysis. An inherent advantage of the in vivo chemical biology approach is the ability to move immediately to other animal species that are more experimentally tractable to explore such possibilities. The suppression by MW01-070C of the human A 3 induced increase in proinflammatory cytokines is especially interesting from a clinical and mechanistic perspective. For example, IL-1 $\beta$ is a glia-derived cytokine found at increased levels in $A D$ pathology [5]. In addition, IL-1 $\beta$ increases production of the amyloid precursor protein [26], further enhances astrocyte activation [19], and brings about increased tau phosphorylation [12]. The suppression of IL-1 $\beta$ suggests one key pathway through which aminopyridazines could exert a therapeutic effect with later interventions.

Suppression of neuroinflammation by MW01-070C correlated with a reduction in neuron loss, synaptic damage, and amyloid plaque deposition in the hippocampus. The mechanism by which MW01-070C treatment leads to neuroprotection is not known. All available data is consistent with neuronal protection being an indirect effect of suppressing glial inflammation, thereby disrupting the neuroinflammatory cycle. However, other interpretations are possible. It is possible, for example, that the compound acts directly on neurons, although we have no evidence to date to support this possibility and MW01-070C has not exhibited in vivo neuroprotective effects in assays requiring a direct neuronal effect. For example, in a hypoxia-ischemia rodent model of neuronal injury where aminopyridazine inhibitors of neuronal apoptosis confer protection [33] but anti-neuroinflammatory compounds do not, MW01-070C is not effective. In addition, in preliminary experiments with neuronal cell cultures, we have not seen any direct protective effects of the compound. The most likely mechanism based on the developmental history of MW01-070C and biological data is that the neuroprotective effect of suppressing neuroinflammation results from a combination of diminishing the direct neurotoxic effects of glial products as well as decreasing downstream neurotoxic effects of $A \beta$ aggregates on neurons. The interesting results with alteration of amyloid plaque deposition with therapeutic intervention are consistent with the latter possibility, and not unexpected. For example, a number of glial proteins co-deposit with and influence $\mathrm{A} \beta$ deposition [1]. It is reasonable to speculate that MW01$070 \mathrm{C}$ might alter $A \beta$ deposition or aggregation via suppression of such glial proteins. It is also theoretically possible that MW01-070C might inhibit aggregation or deposition through direct effects on the $A \beta$ peptide, but no data to date are consistent with this possibility and preliminary in vitro experiments did not reveal obvious effects of MW01-070C on $A \beta$ aggregation. Certainly, the effects of MW01-070C are independent of $A \beta$ processing as human $A \beta$ is infused, not APP.

An attractive feature of the mouse assay described here is that it exhibits features reminiscent of the human disease, and it has potential to facilitate future investigations of mechanism and combinations of disease-relevant processes. Especially important is the robust neuroinflammation and significant neuron damage and loss, a feature not generally seen routinely in transgenic mouse models. The model described here does not show neurofibrillary pathology, but intracerebroventricular infusion of human $A \beta 1-42$ into one of the tau-transgenic mouse models would potentially allow the analysis of animals that have both the $A \beta$ and tau-related pathologies. Animal models used for research focused on discovery of new therapeutic approaches must allow identification of potential therapeutic interventions, but do not need to recapitulate all aspects of the human disease in order to be useful. However, the controlled incorporation of additional disease parameters is especially attractive as it may reveal compounds with dual functions that are desired therapeutically, but can be readily missed in the highly focused assays done in contemporary drug discovery where single targets or selected endpoints are used in early stage discovery efforts. In the post-genomic sequence era, identification of discrete molecular targets can be done rapidly by the use of affinity based and proteomics methods after the discovery of a small molecule therapeutic by unbiased approaches. The robustness, speed, and adaptability of the 
mouse assay described here will facilitate in vivo chemical biology investigations using compounds discovered by both unbiased and biased discovery efforts. As is common in animal studies, there is some variability between experiments in the degree of some endpoints, especially potentially indirect and downstream events such as neuronal loss. Within a single experiment, however, there is little inter-animal variability and significant results can be obtained with only 5-8 mice per group. Overall, the mouse brain infusion model is particularly amenable to drug discovery efforts because of the high penetrance of the pathological endpoints $(>90 \%$ of the mice develop pathology), experimental control over $A \beta$ administration with partial uncoupling of $A \beta$ plaque deposition from APP processing (human $A \beta$ was infused, not APP), the potential for facile use with diverse transgenic animals, and the short time period needed for development of disease-relevant pathology.

In summary, we have shown that a bioavailable inhibitor of glial activation with a novel action different from, and glial inhibitory activity more potent than, currently available NSAIDs can modulate neuroinflammation and plaque deposition in an AD-relevant mouse model of neuroinflammation. An accumulating body of evidence is consistent with the in vivo suppression of neuroinflammation resulting in attenuation of neuronal damage or loss. Taken in its entirety, the data indicate that targeting neuroinflammation pathways with aminopyridazines might provide unique and effective suppression of $\mathrm{AD}$ pathogenic mechanisms, thereby modulating disease progression.

\section{Acknowledgments}

These studies were supported in part by research grants from the Institute for the Study of Aging, the Alzheimer's Association, and NIH (AG13939, AG20243, AG21184, AG10685, NS47586); NIH training grant T32 AG00260; and a research fellowship from the PhRMA Foundation. We thank Catherine Stephens, Carolyn Smith, and Magdalena Zasadzki for technical assistance, and Drs. Mark Wainwright and Anastasia Velentza for helpful discussions and advice.

\section{References}

[1] Akiyama H, Barger S, Barnum S, Bradt B, Bauer J, Cole GM, et al. Inflammation and Alzheimer's disease. Neurobiol Aging 2000;21: 383-421.

[2] Chitturi S, George J. Hepatotoxicity of commonly used drugs: nonsteroidal anti-inflammatory drugs, anti-hypertensives, antidiabetic agents, anticonvulsants, lipid-lowering agents, psychotropic drugs. Semin Liver Dis 2002;22:169-83.

[3] Choi DW. Exploratory clinical testing of neuroscience drugs. Nat Neurosci 2002;5:1023-5.

[4] Dahlgren KN, Manelli AM, Stine B, Baker LK, Krafft GA, LaDu MJ. Oligomeric and fibrillar species of amyloid- $\beta$ peptides differentially affect neuronal viability. J Biol Chem 2002;277:32046-53.
[5] Ehl C, Kolsch H, Ptok U, Jessen F, Schmitz S, Frahnert C, et al. Association of an interleukin-1 beta gene polymorphism at position-511 with Alzheimer's disease. Int J Mol Med 2003;11:2358.

[6] Frautschy SA, Hu W, Kim P, Miller SA, Chu T, Harris-White ME, et al. Phenolic anti-inflammatory antioxidant reversal of A $\beta$-induced cognitive deficits and neuropathology. Neurobiol Aging 2001;22:9931005.

[7] Frautschy SA, Yang F, Calderon L, Cole GM. Rodent models of Alzheimer's disease: rat $A \beta$ infusion approaches to amyloid deposits. Neurobiol Aging 1996;17:311-21.

[8] Griffin WST, Sheng JG, Royston MC, Gentleman SM, McKenzie JE, Graham DI, et al. Glial-neuronal interactions in Alzheimer's disease: the potential role of a "cytokine cycle" in disease progression. Brain Pathol 1998;8:65-72.

[9] Jantzen PT, Connor KE, DiCarlo G, Wenk GL, Wallace JL, Rojiani $\mathrm{AM}$, et al. Microglial activation and $\beta$-amyloid deposit reduction caused by nitric oxide-releasing nonsteroidal anti-inflammatory drug in amyloid precursor protein plus presenilin-1 transgenic mice. J Neurosci 2002;22:2246-54.

[10] Launer LJ. Nonsteroidal anti-inflammatory drugs and Alzheimer's disease. What's next? JAMA 2003;289:2865-7.

[11] Lee WM. Medical progress: drug-induced hepatotoxicity. New Engl J Med 2003;349:474-85.

[12] Li Y, Liu L, Barger SW, Griffin WST. Interleukin-1 mediates pathological effects of microglia on tau phosphorylation and on synaptophysin synthesis in cortical neurons through a p38-MAPK pathway. J Neurosci 2003;23:1605-11.

[13] Lim GP, Yang F, Chu T, Chen P, Beech W, Teter B, et al. Ibuprofen suppresses plaque pathology and inflammation in a mouse model for Alzheimer's disease. J Neurosci 2000;20:5709-14.

[14] Manley PW, Martiny-Baron G, Schlaeppi J-M, Wood J. Therapies directed at vascular endothelial growth factor. Expert Opin Investig Drugs 2002;11:1715-36.

[15] Migaud M, Charlesworth P, Dempster M, Webster LC, Watabe AM, Makhinson M, et al. Enhanced long-term potentiation and impaired learning in mice with mutant postsynaptic density-95 protein. Nature 1998;396:433-9.

[16] Mirzoeva S, Koppal T, Petrova TV, Lukas TJ, Watterson DM, Van Eldik LJ. Screening in a cell-based assay for inhibitors of microglial nitric oxide production reveals calmodulin-regulated protein kinases as potential drug discovery targets. Brain Res 1999;844:12634.

[17] Mirzoeva S, Sawkar A, Zasadzki M, Guo L, Velentza AV, Dunlap $\mathrm{V}$, et al. Discovery of a 3-amino-6-phenyl-pyridazine derivative as a new synthetic antineuroinflammatory compound. J Med Chem 2002;45:563-6.

[18] Morganti-Kossmann MC, Rancan M, Otto VI, Stahel PF, Kossmann T. Role of cerebral inflammation after traumatic brain injury: a revisited concept. Shock 2001;16:165-7.

[19] Mrak RE, Griffin WST. Interleukin-1, neuroinflammation, and Alzheimer's disease. Neurobiol Aging 2001;22:903-8.

[20] Mrak RE, Griffin WST. The role of activated astrocytes and of the neurotrophic cytokine S100B in the pathogenesis of Alzheimer's disease. Neurobiol Aging 2001;22:915-22.

[21] Nathan C. Points of control in inflammation. Nature 2002;420:84652.

[22] Orr CF, Rowe DB, Halliday GM. An inflammatory review of Parkinson's disease. Prog Neurobiol 2002;68:325-40.

[23] Pasinetti GM. From epidemiology to therapeutic trials with antiinflammatory drugs in Alzheimer's disease: the role of NSAIDS and cyclooxygenase in $\beta$-amyloidosis and clinical dementia. J Alzheimer Dis 2002;4:435-45.

[24] Peskind ER, Griffin ST, Akama KT, Raskind MA, Van Eldik LJ. Cerebrospinal fluid S100B is elevated in the earlier stages of Alzheimer's disease. Neurochem Int 2001;39:409-13. 
[25] Petzold A, Jenkins R, Watt HC, Green AJE, Thompson EJ, Keir $\mathrm{G}$, et al. Cerebrospinal fluid S100B correlates with brain atrophy in Alzheimer's disease. Neurosci Lett 2003;336:167-70

[26] Rogers JT, Leiter LM, McPhee J, Cahill CM, Zhan S-S, Potter $\mathrm{H}$, et al. Translation of the Alzheimer amyloid precursor protein mRNA is up-regulated by interleukin-1 through $5^{\prime}$-untranslated region sequences. J Biol Chem 1999;274:6421-31.

[27] Schlaf G, Salje C, Wetter A, Stuertz K, Felgenhauer K, Mader M. Determination of synapsin I and synaptophysin in body fluid by two-site enzyme-linked immunosorbent assays. J Immun Method 1998;213:191-9.

[28] Scorer CA. Preclinical and clinical challenges in the development of disease-modifying therapies for Alzheimer's disease. Drug Discov Today 2001;6:1207-19.

[29] Spinella PC, Dominguez T, Drott HR, Huh J, McCormick L, Rajendra A, et al. S-100ß protein-serum levels in healthy children and its association with outcome in pediatric traumatic brain injury. Crit Care Med 2003;31:939-45.

[30] Stroke Therapy Academic Industry Roundtable (STAIR). Recommendations for standards regarding preclinical neuroprotective and restorative drug development. Stroke 1999;30:2752-758.
[31] Van Eldik LJ, Griffin WST. S100 $\beta$ expression in Alzheimer's disease: relation to neuropathology in brain regions. Biochim Biophys Acta 1994;1223:398-403.

[32] Van Eldik LJ, Wainwright MS. The Janus face of glial-derived S100B: beneficial and detrimental functions in the brain. Restor Neurol Neurosci 2003;21:97-108.

[33] Velentza AV, Wainwright MS, Zasadzki M, Mirzoeva S, Schumacher AM, Haiech J, et al. An aminopyridazine based inhibitor of a proapoptotic protein kinase attenuates hypoxia-ischemia-induced brain damage. Biorg Med Chem Lett 2003;13:3465-70.

[34] Wainwright MS, Rossi J, Schavocky J, Crawford S, Steinhorn $\mathrm{D}$, Velentza AV, et al. Protein kinase involved in lung injury susceptibility: evidence from enzyme isoform genetic knockout and in vivo inhibitor treatment. Proc Natl Acad Sci USA 2003;100:62338 .

[35] Watterson DM, Haiech J, Van Eldik LJ. Discovery of new chemical classes of synthetic ligands that suppress neuroinflammatory responses. J Molecul Neurosci 2002;19:89-93.

[36] Wermuth C-G. Search for new lead compounds: the example of the chemical and pharmacological dissection of aminopyridazines. J Heterocyclic Chem 1998;35:1091-100. 